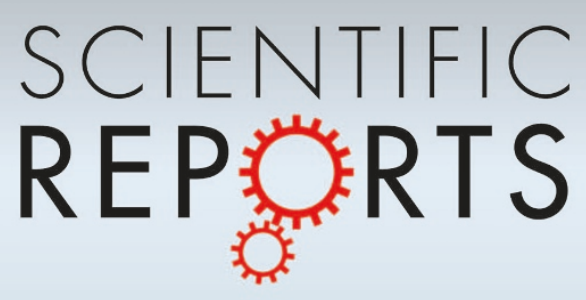

DOI: 10.1038/srep01442

SUBJECT AREAS:

DEVELOPMENT OF THE

NERVOUS SYSTEM

VISUAL SYSTEM

SYNAPTIC TRANSMISSION

NEUROSCIENCE

SCIENTIFIC REPORTS:

$1: 203$

DOI: $10.1038 /$ srep00203

(2011)

Published:

21 December 2011

Updated:

2 April 2013

\section{ERRATUM: The regulatory role of long-term depression in juvenile and adult} mouse ocular dominance plasticity

Kaiyun Yang, Wei Xiong, Guang Yang, Luba Kojic, Changiz Taghibiglou, Yu Tian Wang \& Max Cynader

Brain Research Centre, Vancouver Coastal Health Research Institute and University of British Columbia, Vancouver, BC, Canada V6T $2 \mathrm{~B} 5$.

The authors have noticed that in the original version of this Article, the author Changiz Taghibiglou was omitted from the author list. This has now been corrected and the Author Contributions statement has been updated in both the HTML and PDF versions of the Article. Furthermore, due to a technical error, Figure 5 was published incorrectly in the HTML version of this Article. This has now been corrected online. 